\title{
Entrepreneuriat social et performance économique : le cas des organisations de producteurs de la vallée du fleuve Sénégal
}

\author{
Ahmadou Aly Mbaye ${ }^{a}$, Fatou Gueye ${ }^{b}$
}

RÉSUMÉ. Dans cet article, nous partons d'une étude de cas sur les modèles d'organisation de la production de riz dans la vallée du fleuve Sénégal pour montrer comment les performances productives des riziculteurs sont influencées par le modèle coopératif en vigueur dans la vallée. Le modèle d'entrepreneuriat social que nous préconisons correspond à un arrangement institutionnel offrant plus de résilience aux producteurs visà-vis des chocs climatiques et sanitaire que ceux en vigueur dans les autres zones de production.

ABSTRACT. In this paper, we start with a case study of rice production organization models in the Senegal River V alley to show how the productive performance of rice farmers is influenced by the cooperative model in force in the valley. The model of social entrepreneurship that we recommend corresponds to an institutional arrangement that offers greater resilience to and climate and sanitary shocks to producers than those in other production areas.

\section{Introduction}

Au Sénégal, comme dans d'autres pays d'Afrique de l'Ouest, l'agriculture est entièrement structurée par le secteur informel. Dans leur étude sur le Bénin, le Burkina Faso et le Sénégal, les chercheurs Benjamin et Mbaye (2012) ainsi que Mbaye, Golub et Gueye (2020) concluent que le secteur agricole est le secteur le plus touché par l'informalité. Les principales caractéristiques de l'économie informelle sont la précarité des emplois existants et la faiblesse des revenus. Les conventions collectives du secteur agricole correspondent aux niveaux les plus bas de rémunération et de protection des salariés. Par exemple, au Sénégal, le salaire minimum agricole garanti (SMAG) est fixé à 182,95 francs $\mathrm{CFA} /$ heure $(0,28 €$ ou $0,43 \$ \mathrm{CA})$ par le Décret no 96-154 du 19 février 1996 fixant les salaires minima interprofessionnels et agricoles garantis, alors qu'il dépasse le double pour les métiers régis par d'autres conventions collectives. Ce SMAG n'a pas changé depuis 25 ans.
Par ailleurs, l'emploi salarié représente une très faible part de l'emploi dans les zones rurales. Également informels, le travail indépendant et le travail domestique correspondent à des niveaux de rémunération et de protection précaires.

Une des particularités du secteur agricole en Afrique de l'Ouest est son hétérogénéité, avec des organisations paysannes sophistiquées qui régissent certaines chaînes de valeur (p. ex., le riz au Sénégal), tandis que des pratiques agricoles et des niveaux d'organisation plus traditionnels sont observés pour d'autres produits agricoles. Ces formes d'organisation des producteurs, qui font souvent partie intégrante des mécanismes de soutien de l'État aux producteurs de riz, ne se retrouvent pas forcément dans les zones de production d'autres produits agricoles. L'objectif de cet article est de montrer comment les modèles d'organisation de la zone de production de riz et d'autres zones de production agricole affectent la dynamique de l'emploi et de la formation des revenus.

\footnotetext{
${ }^{a}$ Recteur, Université Cheikh Anta Diop, Dakar

${ }^{\mathrm{b}}$ Professeure, directrice du Laboratoire d'Analyse des Politiques de Développement, Univeresité Cheikh Anta Diop, Dakar
} 
Près de la moitié de la population sénégalaise est âgée de moins de 20 ans. Chaque année, la population en âge de travailler n'augmente que d'environ 250000 personnes, tandis que l'économie formelle ne crée qu'environ 50000 emplois (Golub et Mbaye, 2015). Cela crée de fortes tensions sur le marché du travail, où la somme du taux de chômage et du taux de sous-emploi n'est pas très éloignée de $50 \%$ de la population active totale. Le dernier recensement de la population et de l'habitat au Sénégal, qui date de 2013 montre que la population en âge de travailler, entre 15 et 64 ans, est de 7,3 millions de personnes, alors que la population active est estimée à 3,1 millions de personnes, soit environ $32 \%$ de la population totale. La population en âge de travailler se répartit entre les zones rurales $(59 \%)$ et les zones urbaines $(41 \%)$, et les caractéristiques de l'emploi sont très différentes entre ces deux zones. Selon l'Enquête sénégalaise auprès des ménages (ESAM; République du Sénégal, 2001), les principales caractéristiques de l'emploi rural au pays sont les suivantes :

- Le nombre d'années de travail est plus élevé que dans les villes. Les jeunes commencent à travailler avant l'âge de 15 ans (près de 1 enfant sur 2) et les travailleurs plus âgés travaillent au-delà de 64 ans (près de 9 personnes âgées sur 10);

- L'emploi est concentré autour du secteur primaire dans les petites exploitations agricoles. Environ 8 travailleurs sur 10 ont l'agriculture, l'élevage ou l'exploitation forestière comme principale source de revenus. Les exploitations agricoles dans lesquelles la main-d'œuvre est employée sont de type familial ou individuel, c'est-à-dire des petites entreprises ;

- Les conditions d'emploi sont extrêmement précaires. Si les taux d'emploi sont en moyenne plus élevés dans les campagnes, la majorité des travailleurs sont des travailleurs saisonniers (6 sur 10) et seul un quart d'entre eux considère avoir un emploi permanent à temps plein. Le travail informel est presque universel, car la quasi-totalité des travailleurs ne bénéficie pas d'une couverture sociale formelle ;

- Enfin, le niveau d'éducation de ceux qui travaillent est très faible. Plus de 7 personnes (de plus de 15 ans) sur 10 n'ont pas été scolarisées.

Dans les zones rurales, le marché du travail comprend essentiellement deux groupes d'activités : les activités agricoles et les activités non agricoles.
Dans les deux cas, l'offre de main-d'œuvre dépend de plusieurs facteurs, dont la démographie, les normes sociales, l'accès à la terre, l'éducation, etc. La demande est exprimée à la fois par les entreprises agricoles et non agricoles, et dépend du système de production, des volumes d'investissement, de l'accès aux marchés, etc.

Le chômage officiel est moins fréquent dans les zones rurales que dans les zones urbaines. Dans les zones rurales, les principaux problèmes liés à l'emploi des jeunes et des femmes sont le sous-emploi et l'inactivité. La proportion de jeunes inactifs est constituée de personnes âgées de 15 à 34 ans qui ne sont ni au travail ni à l'école. Près de la moitié des jeunes et des femmes inactives sont employés dans l'agriculture familiale et le paiement est généralement effectué en nature (nourriture principale et logement). Ces femmes sont souvent appelées « aides familiales résidentes ».

Aux côtés des jeunes inactifs et des aides familiales, on trouve les jeunes chefs d'unités de production agricole (UPA). Ils représentent une faible proportion de la main-d'œuvre et sont majoritairement des hommes, les femmes ne représentant que $29 \%$ des chefs d'UPA. En outre, $88 \%$ des jeunes n'ont pas de source de revenus régulière. Les femmes sont également touchées par cette contrainte, surtout les plus jeunes. C'est la preuve que les jeunes et les femmes ont un accès limité à la terre et au capital.

De même, les jeunes et les femmes sont peu représentés dans les organisations de producteurs (OP). Les femmes, en particulier, dirigent rarement à titre individuel. Elles participent soit par l'intermédiaire de groupes de femmes, soit par l'intermédiaire de leur chef de famille. Le Conseil national de concertation et de coopération des ruraux (CNCR) a créé un collège des jeunes en réponse à certaines de ces préoccupations. Des suggestions ont été faites à l'État pour réserver des quotas aux femmes et aux jeunes en cas de nouveaux projets d'aménagement du territoire (Hathie et collab., 2015).

L'accès aux moyens de production est limité chez les jeunes ruraux, notamment en ce qui concerne la terre et les équipements. En outre, la capacité financière des jeunes est faible, de sorte qu'ils démarrent souvent avec des activités qui exigent un investissement initial faible et un retour rapide (p. ex., l'horticulture et l'agriculture à cycle court). Le manque de soutien financier et le manque 
d'expérience rendent les jeunes plus vulnérables aux incertitudes telles que les fluctuations du marché, les chocs climatiques et le vol (FERT et IECD, 2015).

Le niveau d'éducation de la main-d'œuvre rurale est relativement faible, avec une proportion écrasante de personnes sans instruction. La majorité des personnes instruites n'ont pas dépassé l'ordre primaire. Cependant, l'enseignement coranique est très présent. Ce faible niveau d'éducation touche surtout les femmes; plus de la moitié des femmes rurales n’ont jamais été scolarisées.

La suite de cet article est organisée comme suit : d'abord, nous faisons une analyse de la chaîne de valeur du riz dans le delta du fleuve Sénégal, puis traitons de l'impact des chaînes de valeur sur le potentiel d'emploi et le revenu des organisations paysannes. Enfin, nous analysons, dans le cas de Débi-Tiguet, l'importance de l'innovation sociale et de la performance économique.

\section{La chaîne de valeur du riz dans le delta du fleuve Sénégal}

Les opportunités agricoles dans le delta sont abondantes en raison de la fertilité des sols et de la disponibilité de l'eau. Plusieurs produits sont développés dans cette région, notamment le maïs, le sorgho, la tomate industrielle, les oignons, les patates douces, l'arachide, le coton et le riz. La culture du riz y est de loin l'activité la plus répandue. Le riz est la base de l'alimentation au Sénégal et la production nationale ne couvre que moins de $20 \%$ des besoins totaux. Le reste est importé, principalement d'Asie (voir tableau 1).

\begin{tabular}{lcccccccc}
\hline Utilisations et ressources & $\mathbf{1 9 9 5}$ & $\mathbf{1 9 9 6}$ & $\mathbf{1 9 9 7}$ & $\mathbf{1 9 9 8}$ & $\mathbf{1 9 9 9}$ & $\mathbf{2 0 0 0}$ & $\mathbf{2 0 0 1}$ & $\mathbf{2 0 0 2}$ \\
\hline Populations (équivalent adulte) & 6483645 & 6658703 & 838488 & 7023128 & 7212752 & 7407496 & 7607499 & 7812901 \\
$\begin{array}{l}\text { Consommation } \\
\text { moyenne/an/habitant (kg) }\end{array}$ & 60 & 2 & 63 & 65 & 67 & 69 & 70 & 72 \\
Demande totale en riz (t) & 389019 & 410309 & 432765 & 456450 & 481431 & 507779 & 535569 & 564881 \\
Production totale de riz (t) & 100750 & 96850 & 113100 & 141700 & 138450 & 124103 & 137124 & 158013 \\
Importations (t) & 435500 & 627200 & 452000 & 535300 & 658070 & 501657 & 632253 & 709575 \\
Aide alimentaire en riz (t) & 6765 & 4904 & 8238 & 1997 & 8993 & 6000 & 6000 & 6000 \\
Offre totale de riz (t) & 543015 & 728954 & 573338 & 678997 & 805513 & 631760 & 775377 & 873588 \\
Inventaire (t) & 153996 & 318645 & 140573 & 222547 & 324082 & 123981 & 239808 & 308703 \\
\hline
\end{tabular}

Note. $\mathrm{T}=$ tonne métrique.

Tableau 1 - Utilisations et ressources dud riz au Sénégal (1995-2002)

Source : SAED, 2003

La contrainte majeure pour la culture du riz au Sénégal est le niveau d'utilisation des semences certifiées. La plupart des semences utilisées proviennent des cultures de l'année précédente. Elles sont donc moins chères, mais aussi moins efficaces que les semences certifiées. Cependant, l'utilisation des semences certifiées a augmenté très rapidement ces derniers temps, passant de 634 tonnes en 1994 à 2250 tonnes en 2000. Les rendements ont suivi une tendance similaire grâce à l'intervention des bailleurs de fonds dans la vallée. Or, le principal défi consiste maintenant à rendre la production nationale de riz compétitive par rapport au riz asiatique, qui est beaucoup moins cher, même sur les marchés locaux.
Les enquêtes sur les ménages ruraux (EJMAO : Emploi des Jeunes et Migration en Afrique de l'Ouest menées par l'Initiative prospective agricole et rurale (IPAR; Mbaye et collab., 2016), ont montré que $91 \%$ des ménages ruraux sont employés dans le secteur agricole. Les autres ménages $(9 \%)$ sont exclusivement engagés dans des activités non agricoles. Cette répartition est très hétérogène entre les zones de production. Par exemple, dans le delta, qui est la principale zone de production de riz, il est possible de produire toute l'année pour $52 \%$ des ménages exclusivement agricoles, pour $4 \%$ des ménages non agricoles et pour $33 \%$ des ménages mixtes. Cela peut être comparé à d'autres zones telles que le Bassin 
arachidier, où $25 \%$ des ménages sont exclusivement agricoles, $7 \%$ sont des ménages non agricoles et $68 \%$ sont des ménages mixtes.

Au Sénégal, il existe deux systèmes de production de riz : irrigué et pluvial. Le riz pluvial couvre 78000 ha et fournit 150000 tonnes de paddy (riz non décortiqué), soit $30 \%$ de la production nationale. Selon le Programme national d'autosuffisance en riz (PNAR, 2009), la production annuelle moyenne nationale est de 500000 à 600000 tonnes de paddy. Les investissements réalisés par le gouvernement sénégalais pour contrôler l'eau ont permis de stimuler la production de paddy.

Dans le delta, les installations publiques sont occupées par d'anciens groupes de producteurs convertis en groupements d'intérêt économique (GIE) ou en sections villageoises, puis regroupés en UPA pour gérer des installations communes telles que des stations de pompage, des magasins et des rizeries.
À la suite de la crise économique mondiale de 2008, le prix du riz a augmenté de 50 à $100 \%$ en Afrique de l'Ouest. Le prix mondial du Thaï 25 , la variété de riz la plus importée par le Sénégal, a presque triplé à la mi-2008 (FAO, 2009). En avril 2008, au début de la crise, le Sénégal a lancé la Grande offensive agricole pour la nourriture et l'abondance (GOANA). Dans le cadre de ce programme, des investissements massifs dans la filière locale du riz ont été réalisés avec l'objectif ambitieux d'atteindre l'autosuffisance alimentaire d'ici 2015.

Plusieurs acteurs interviennent dans le circuit de commercialisation (voir figure 1). Les collecteurs, les grossistes, les semi-grossistes et les détaillants interviennent après la production. Ces catégories sont différenciées en fonction de la capacité financière, du volume des ventes et de la mobilité. Situés dans les centres urbains, les grossistes sont approvisionnés par les collecteurs.

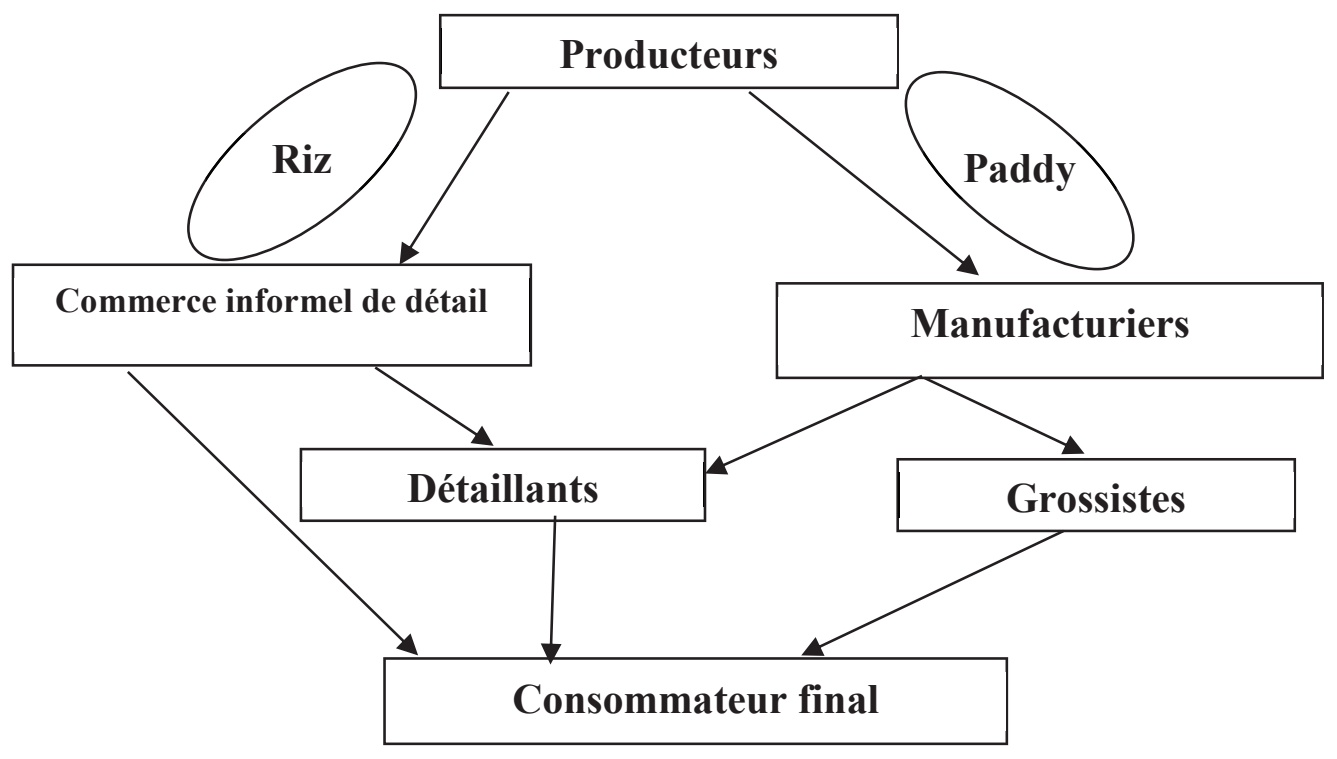

Figure 1 - Circuit de commercialisation du riz Source : Fall et Cissé, 2010

\section{Le système de distribution et de traitement}

Le riz commercialisé localement est souvent produit à partir de rizières irriguées, alors que le riz pluvial est principalement destiné à l'autoconsommation. Plusieurs acteurs interviennent dans la commercialisation du riz dans la vallée du fleuve et dans le bassin de l'Anambé. La transformation est effectuée par les producteurs eux-mêmes ou par des prestataires de services dont l'activité principale est la transformation.

Les unités de transformation peuvent être divisées en trois groupes dans les zones de production irriguées. Il y a les décortiqueurs villageois, qui transforment 75 à $80 \%$ du produit national. Ces décortiqueurs ont une faible capacité : ils ne dépassent pas 100 sacs par jour. Cependant, 343 unités, 
soit $93 \%$ des unités de transformation, sont assez importantes dans la zone irriguée et représentent une capacité de transformation de 160000 tonnes (SAED, 2011). En outre, il existe une transformation semi-industrielle et industrielle, qui transforme 20 à $25 \%$ de la production nationale. Elle comprend 41 unités, dont 26 sont fonctionnelles et produisent du riz de meilleure qualité, selon les normes requises. Toutefois, certains producteurs effectuent leur propre transformation et commercialisation.

\section{Innovations adoptées et impact sur les rendements}

Les interventions de gestion intégrée des cultures de riz (GICR) dans toute la vallée, avec l'utilisation de variétés améliorées, ont contribué à augmenter les rendements de 3,8 à 5,5 t/ha (Defoer et collab., 2004). Les modèles de croissance du riz prévoient un rendement potentiel de 9 à 12 t/ha de riz irrigué au Sahel (Dingkuhn et Sow, 1997). Cela a été confirmé à l'échelle des exploitations agricoles, où des rendements de $12 \mathrm{t} /$ ha ont été signalés pendant la saison chaude et sèche (Wopereis et collab., 1999) et même pendant la saison des pluies par certains producteurs interrogés dans le cadre de cette étude. Malgré cette performance, l'écart entre les rendements fermes et potentiels reste important.

\section{L'impact des chaînes de valeur sur l'emploi et la création de revenus : comparaison entre la zone productrice de riz et les zones non productrices de riz}

Dans cette section, nous analysons l'impact des chaînes de valeur sur le revenu et l'emploi au Sénégal. Les données utilisées dans cette section proviennent de la base de données d'Emploi des jeunes et migration en Afrique de l'Ouest (EJMAO; Hathie et collab., 2015; Mbaye et collab., 2016).
Elles ont été collectées en 2013 par le biais d'Enquêtes sénégalaises auprès des ménages (ESAM). Ces enquêtes ont permis de recueillir des informations sur 1500 ménages dans le Bassin arachidier, dans le delta du fleuve Sénégal et dans les Niayes. Dans ces trois zones agroclimatiques, il y a eu un choix raisonné de villages sur la base d'une différenciation économique et sociologique. Les ménages de chaque village ont ensuite été tirés au sort. La zone du delta du fleuve contient l'essentiel de la culture irriguée du riz, du transport et de la transformation au Sénégal, alors que d'autres zones produisent principalement d'autres cultures, comme les légumes, l'arachide et les céréales.

Le type d'organisation observé pour le riz dans le delta est très différent de celui des autres zones de production agricole : un écosystème de production de riz principalement organisé autour d'une chaîne de valeur claire, avec une répartition claire des fonctions sur des activités diverses et verticalement intégrées. Pour d'autres types de cultures, une telle organisation n'existe pas. La comparaison des deux types de zones est donc susceptible de mettre en lumière la relation entre les chaînes de valeur et les niveaux/modèles de revenu et d'emploi.

\subsection{Caractéristiques sociodémographiques du travail et des ménages}

Il n'est pas surprenant que le sexe ne diffère pas beaucoup dans les deux types de zones (voir figure 2 et tableau 2). La répartition par sexe de la maind'œuvre rurale montre que les femmes sont plus actives sur le marché du travail dans la zone de production de riz $(52,3 \%)$ que dans les autres zones $(48,8 \%)$. Si l'on considère la répartition par sexe et par âge de la main-d'œuvre rurale, les jeunes femmes sont majoritaires tant dans la zone productrice de riz que dans les autres zones rurales du Sénégal. 




Figure 2 - Part de la population active par sexe

Source : IPAR, 2015 et calcul des auteurs

\begin{tabular}{lcccc}
\hline \multirow{2}{*}{ Groupe d'âge } & \multicolumn{2}{c}{ Zone de production de riz } & \multicolumn{2}{c}{ Autres zones } \\
& Homme & Femme & Homme & Femme \\
\hline $10-14$ ans & $14,7 \%$ & $16,6 \%$ & $20,20 \%$ & $17,27 \%$ \\
$15-19$ ans & $20,0 \%$ & $15,2 \%$ & $18,74 \%$ & $17,21 \%$ \\
$20-24$ ans & $12,1 \%$ & $12,3 \%$ & $11,59 \%$ & $12,56 \%$ \\
$25-29$ ans & $9,1 \%$ & $9,5 \%$ & $8,82 \%$ & $11,29 \%$ \\
$30-34$ ans & $8,4 \%$ & $9,2 \%$ & $5,42 \%$ & $9,49 \%$ \\
$35-39$ ans & $7,2 \%$ & $10,0 \%$ & $4,50 \%$ & $7,36 \%$ \\
$40-44$ ans & $6,7 \%$ & $8,1 \%$ & $4,80 \%$ & $5,71 \%$ \\
$45-49$ ans & $5,6 \%$ & $6,0 \%$ & $4,52 \%$ & $4,97 \%$ \\
$50-54$ ans & $4,9 \%$ & $5,7 \%$ & $3,34 \%$ & $5,11 \%$ \\
$55-59$ ans & $3,3 \%$ & $3,0 \%$ & $3,91 \%$ & $2,95 \%$ \\
$60-64$ ans & $3,7 \%$ & $2,6 \%$ & $2,76 \%$ & $2,50 \%$ \\
$65-69$ ans & $2,0 \%$ & $0,8 \%$ & $2,11 \%$ & $1,15 \%$ \\
$70-74$ ans & $1,2 \%$ & $0,3 \%$ & $1,07 \%$ & $1,28 \%$ \\
$75-79$ ans & $0,4 \%$ & $0,3 \%$ & $0,71 \%$ & $0,68 \%$ \\
$80-84$ ans & $0,5 \%$ & $0,3 \%$ & $0,59 \%$ & $0,25 \%$ \\
85 ans - & $0,2 \%$ & $0,1 \%$ & $0,17 \%$ & $0,22 \%$
\end{tabular}

Tableau 2 - Part de la population active par sexe et par âge

Source : IPAR, 2015 et calcul des auteurs

Lorsque l'on considère le niveau d'éducation de la population active, on constate que la majeure partie de la population active rurale dans la production de riz a un niveau d'éducation qui ne dépasse pas l'ordre primaire (34,7\% des hommes et $41,3 \%$ des femmes). Cependant, il est beaucoup plus élevé que dans les zones non productrices de riz: $15 \%$ de la main- d'œuvre rurale dans la zone de production de riz a un niveau d'éducation élémentaire moyen, contre seulement $7 \%$ dans les autres zones. Il en va de même pour le niveau d'éducation secondaire, qui comprend 3,8\% de la main-d'œuvre dans la zone de production de riz et seulement $0,05 \%$ de la main-d'œuvre dans les autres zones (voir figure 3). 


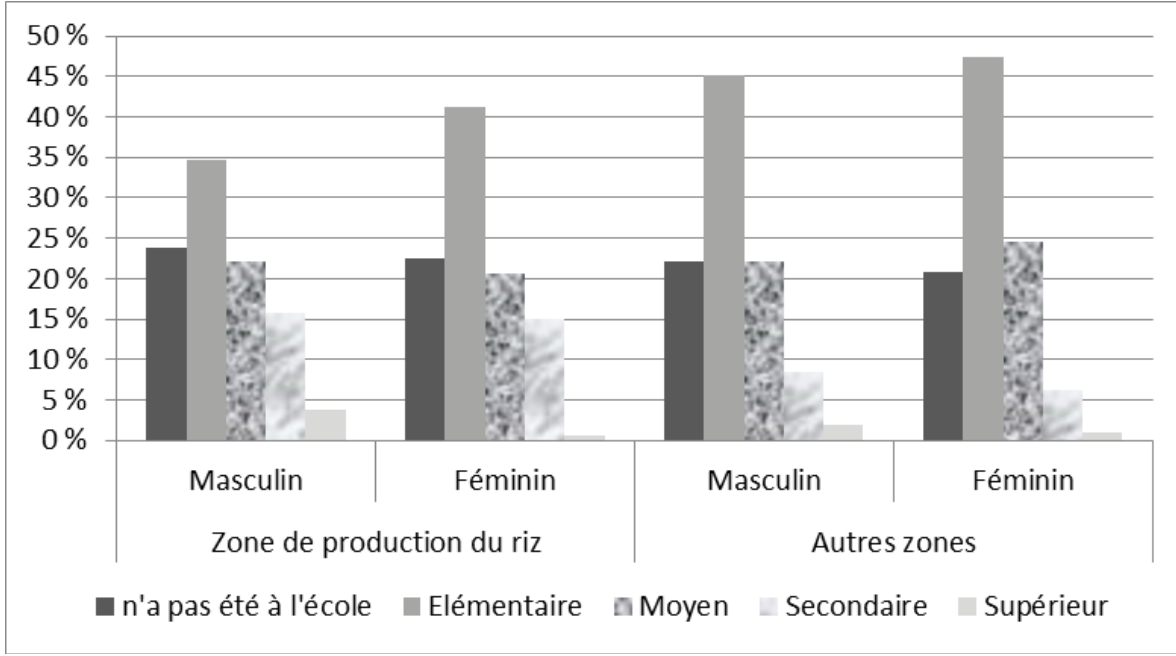

Figure 3 - Niveau d'éducation de la main-d'œuvre rurale Source : IPAR, 2015 et calcul des auteurs

La taille des ménages est une autre caractéristique distinctive de ces données: les ménages du delta du fleuve Sénégal sont légèrement plus petits qu'ailleurs (voir tableau 3). Par ailleurs, l'âge moyen du chef de ménage est de 50 ans dans la zone de production de riz, contre 53 ans pour les autres zones. Cependant, c'est dans la zone de production de riz qu'on trouve la plus forte probabilité de chefs de ménage masculins $(95 \%)$, contre $89 \%$ dans les autres zones.

\begin{tabular}{lccc} 
Caractéristiques & $\begin{array}{c}\text { Zone de } \\
\text { production de riz }\end{array}$ & $\begin{array}{c}\text { Autres } \\
\text { zones }\end{array}$ & Total \\
\hline Taille moyenne des ménages & 9,4 & 10,7 & 10,8 \\
Nbre moyen de personnes de + de 10 ans dans le ménage & 6,8 & 7,45 & 7,5 \\
$\%$ de chefs de famille masculins & $95 \%$ & $89 \%$ & $90 \%$ \\
$\%$ de chefs de famille féminins & $5 \%$ & $12 \%$ & $10 \%$ \\
Âge moyen du chef de ménage & 50,5 ans & 53,8 ans & 53,2 ans \\
\hline
\end{tabular}

Tableau 3 - Caractéristiques sociodémographiques des ménages ruraux Source : IPAR, 2015 et calcul des auteurs

La structure de l'âge de la main-d'œuvre ne diffère pas de manière significative entre la zone de production de riz et les autres zones. Les jeunes (15-34 ans) représentent $48,9 \%$ de la main- d'œuvre dans la production de riz et 49,8\% dans les autres zones (voir figure 4). De même, c'est dans la zone de production de riz que la plupart des ménages ont plus d'une UPA (voir figure 5). 


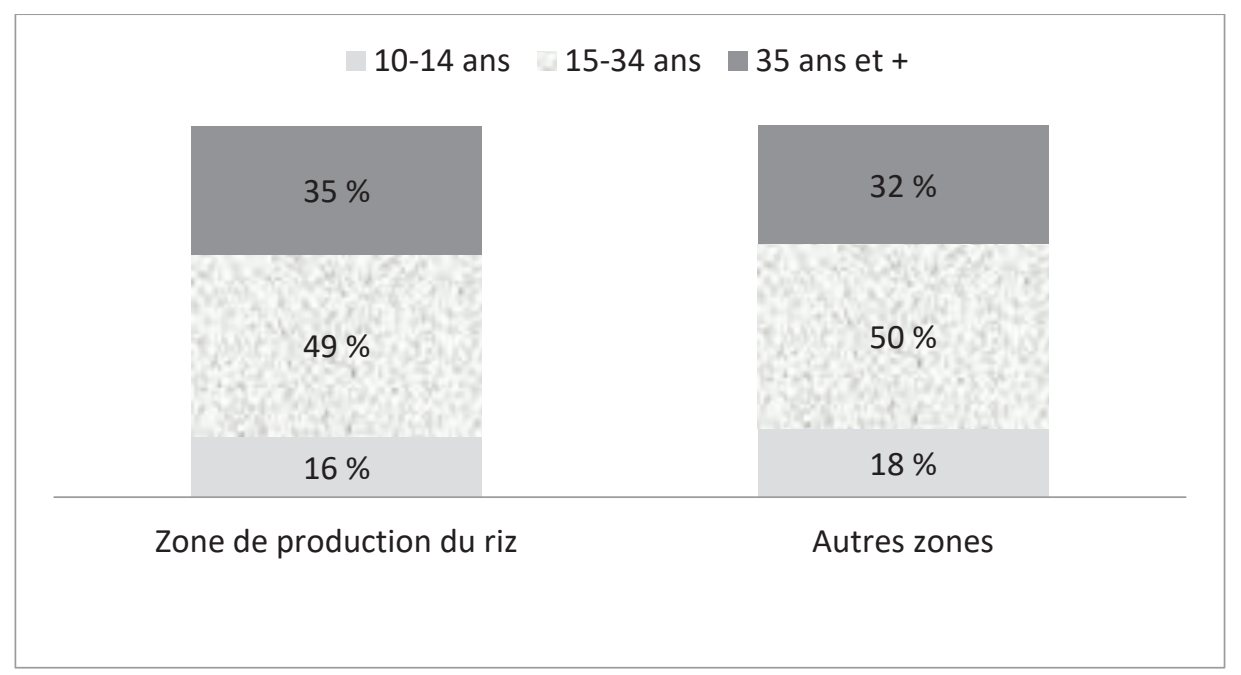

Figure 4 - Structure par âge de la population en âge de travailler Source : IPAR, 2015 et calcul des auteurs



Figure 5 - Répartition du nombre d'unités de production agricole (UPA) par zone Source : IPAR, 2015 et calcul des auteurs

Une grande majorité des travailleurs familiaux travaillent en permanence sur l'exploitation familiale, tant dans la production de riz que dans les autres zones (voir figure 6). Cependant, une grande partie de la main-d'œuvre externe employée dans les exploitations familiales de la zone de production de riz provient d'autres régions agroécologiques par le biais de la migration interne $(58,17 \%)$. Dans les autres zones, en revanche, la main-d'œuvre externe provient principalement du même village $(67,73 \%)$ (voir tableau 4$)$. 


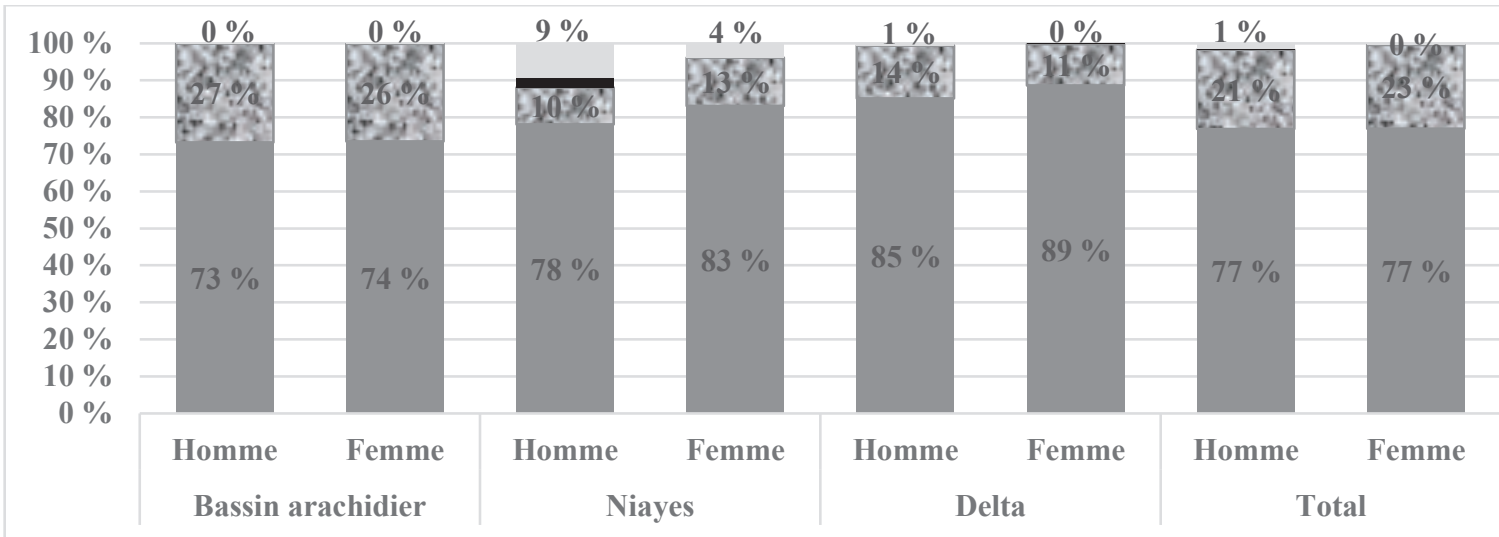

- Main d'oeuvre familiale permanente $\square$ Main d'oeuvre familaile saisonnière

- Employé permanent

Employé saisonnier

Figure 6 - Situation de la main-d'œuvre familiale dans les exploitations agricoles Source : IPAR, 2015 et calcul des auteurs

\begin{tabular}{lcc}
\hline Origine & Zone de production de riz & Autres zones \\
\hline Même village & $30,57 \%$ & $67,73 \%$ \\
Village voisin & $10,19 \%$ & $14,32 \%$ \\
Migration & $58,17 \%$ & $16,66 \%$ \\
Non disponible & $1,06 \%$ & $1,30 \%$ \\
\hline
\end{tabular}

Tableau 4 - Origine de la main-d'œuvre externe

Source : IPAR, 2015 et calcul des auteurs

En résumé, hormis le niveau d'éducation, les caractéristiques sociodémographiques des ménages des deux types de zones semblent similaires.
Quant au taux de dépendance, il est plus faible dans le delta $(43 \%)$ que dans les autres zones $(53 \%)$ (voir tableau 5).

\begin{tabular}{lccc}
\hline & Zone de production de riz & Autres zones & Total \\
\hline $\begin{array}{l}N^{\text {bre }} \text { de personnes occupées par ménage } \\
(10 \text { ans ou }+)\end{array}$ & 3,5 & 5,4 & 5,1 \\
Taux de dépendance & $43 \%$ & $53 \%$ & $49 \%$ \\
\hline
\end{tabular}

Tableau 5 - Quelques indicateurs de la population active rurale Source : IPAR, 2015 et calcul des auteurs

\section{$2.2 \quad$ Structure de l'emploi}

Plusieurs tendances caractérisent l'emploi dans les deux types de zones. Premièrement, de nombreuses activités non agricoles coexistent avec des activités agricoles. Elles englobent le commerce, l'artisanat, le transport et les services. La figure 7 montre la répartition des activités non agricoles par zone. 




Figure 7 - Structure de l'artisanat et du commerce par zone Source : IPAR, 2015 et calcul des auteurs

Il existe une différence en matière de genre dans la pratique de ces activités rurales non agricoles. Les femmes exercent principalement des activités telles que le commerce (vente de produits alimentaires), le service (décorticage des arachides, vannage, etc.), l'artisanat (coiffure, couture) ainsi que la transformation de produits agricoles locaux (huile et beurre d'arachide).

Les hommes sont plus nombreux dans le secteur des transports (voitures, chevaux, etc.) et dans l'artisanat dans une grande variété de métiers, notamment la mécanique, le bâtiment (maçonnerie), la menuiserie, la couture et la cordonnerie. Les femmes dominent les activités non agricoles. Elles représentent $71 \%$ des propriétaires d'unité de production non agricole (UPNA) dans le Bassin arachidier, $63 \%$ dans les Niayes et $49 \%$ dans le delta.

L'attractivité des activités non agricoles pour les femmes peut également s'expliquer par des barrières de toutes natures. La principale caractéristique des activités non agricoles est qu'elles constituent une activité indépendante (travail indépendant) pour la plupart des propriétaires (voir figure 8). En effet, la majorité des chefs d'activités non agricoles (78\% dans le Bassin arachidier, $88 \%$ dans les Niayes et $67 \%$ dans le delta) exercent leurs activités sans salariés ni recours à la main-d'œuvre familiale. 


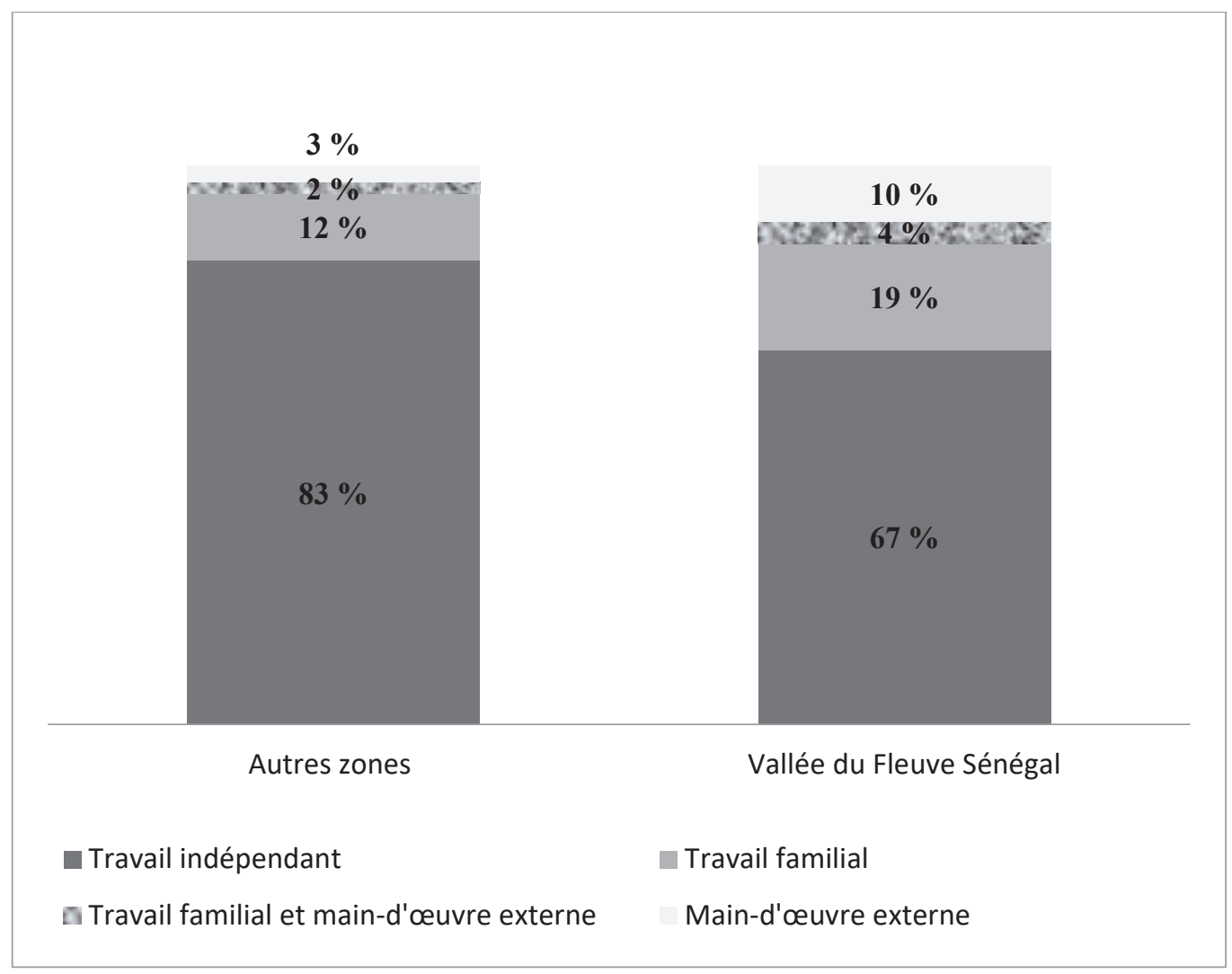

Figure 8 - Répartition des types de main-d'œuvre utilisés par les UPNA Source : IPAR, 2015 et calcul des auteurs

Le système salarial concerne les activités agricoles et non agricoles, et occupe une part relativement faible des actifs dans les trois zones de production agricole. Le salarié agricole est quasiment inexistant dans le Bassin arachidier (0,2\% de la population active). (Sur les 4561 actifs recensés dans le Bassin arachidier, il n'y a que 9 ouvriers agricoles.) Il n'y a pas de main-d'œuvre migrante dans le Bassin arachidier. Les emplois salariés sont relativement peu nombreux dans les Niayes $(3 \%)$ et dans le delta $(2 \%)$.
Les salariés non agricoles détiennent un pourcentage relativement plus élevé de biens dans le delta $(16 \%)$ que dans le Bassin arachidier (9\%) et aux Niayes $(10 \%)$.

La majorité de la main-d'œuvre rurale dans toutes les zones d'étude n'a pas de contrat avec ses employeurs, qu'il s'agisse de son activité principale ou secondaire (voir figure 9). En fait, dans le Bassin arachidier, plus de $80 \%$ des travailleurs ont déclaré n'avoir aucun type de contrat avec leur employeur; ce pourcentage est de $55 \%$ dans les Niayes et de $52 \%$ dans le delta. 




Figure 9- Types de contrats dans l'activité primaire Source : IPAR, 2015 et calcul des auteurs

\section{Le cas du projet Débi-Tiguet}

Les villages de Débi-Tiguet sont situés sur la rive gauche du fleuve Sénégal, à $45 \mathrm{Km}$ en amont (voir figure 10). Avec une superficie de $500 \mathrm{~km}^{2}$ le Débi Irrigation Project (DIP), sous financement du Japon, bénéficie de conditions géographiques favorables, de la qualité du sol et de la disponibilité de l'eau comparativement aux autres cuvettes du delta. Selon Mbaye (2008): " avec un budget total de 8 millions de francs $\mathrm{CFA}^{1}$, le but du projet est de réhabiliter le rayonnage de Débi. Ses principaux objectifs sont les suivants :

- une augmentation de l'autosuffisance alimentaire;

- l'encouragement d'une gestion autonome des exploitations agricoles par les paysans;

- une amélioration des infrastructures hydroagricoles dans les deux villages; et
- une augmentation de la productivité et des revenus des producteurs dans la région par l'introduction d'une double récolte dans l'année. »

Le DIP a permis la construction d'un entrepôt, la mise à disposition de tracteurs et de camions, et la réfection de la route reliant les deux villages. Le projet comporte également un volet d'assistance technique, qui consiste à former les agents de la Société d'aménagement et d'exploitation des terres du delta du fleuve Sénégal et des vallées du fleuve Sénégal et de la Falémé (SAED) à la maitrise des systèmes comptables adaptés à ce type de projet. Les paysans sont également formés pour mettre en œuvre les techniques de double culture et pour préparer les documents nécessaires aux demandes de prêts bancaires. La SAED a été créée en 1965 pour gérer le périmètre d'irrigation de la région. L'organisation est composée de cinq divisions et de quatre délégations. Le DIP est sous la juridiction du Bureau d'études et de contrôle (BEC) et de la délégation de Dagana. 




Légende :

\begin{tabular}{|l|l|l|l|}
\hline GIE Débi II (88,44 ha) & $\vdots$ & Autres sections villageoises \\
\hline & Zones humides & & Autre source d'eau \\
\hline & Station de pompage principale & $\mathbf{0}$ & Répartiteurs \\
\hline $\mathbf{3 3}$ & Terminal des eaux & & \\
\hline Note. Les parcellaires : superficie par section villageoise. & \\
\hline
\end{tabular}

Figure 10 - Situation géographique du DIP Source : Mbaye, 2008

Autrefois, Débi et Tiguet étaient des villages de pêcheurs. Les activités économiques étaient alors rudimentaires. Outre la pêche, elles consistaient en la cueillette et en une agriculture orientée vers l'alimentation. Selon Mbaye (2008), le premier essai d'irrigation dans la région a eu lieu dans le bassin du Djoudj en 1964. Il couvre une superficie de 80 ha et a doublé, pour atteindre 160 ha en 1966. Le Koweït a financé la gestion des terres dans ces villages en 1980 . Une superficie de 737 ha a été couverte dans le cadre de ce programme. Les Japonais ont commencé à financer le projet en 1996, mais la réhabilitation de la cuvette (rack) a commencé en 1992. La superficie des terres gérées est de 982 ha. Les autres zones environnantes sont également favorables à l'irrigation. On estime que 1700 ha de terres irrigables y sont disponibles pour des activités privées.
La principale impression que nous avons eue au cours de nos entretiens avec les bénéficiaires est que le programme a considérablement amélioré le niveau de vie dans les deux villages. Plus important encore, il a amélioré les capacités nationales en matière de gestion des organisations humaines. Avant la mise en œuvre du projet, les modèles d'organisation dans les deux villages ainsi que dans les villages environnants étaient centrés sur les coopératives, et les villages étaient des sections de ces coopératives. L'unité de gestion du projet consiste en une union des organisations de producteurs (OP) de 28 personnes (14 de chaque village) choisies sur une base paritaire. La hiérarchie organisationnelle de la structure de gestion est la suivante (voir figure 11) : 
- L’OP, dont les présidents sont automatiquement membres du conseil d'administration;

- L'assemblée générale, qui est composée de toutes les personnes vivant dans les deux villages, organisée en OP. Elle se réunit deux fois par an : au début et à la fin du cycle de production. Elle nomme les membres du conseil d'administration;

- Le conseil d'administration, qui est composé de 28 personnes (14 par village) choisies sur une base paritaire. Il se réunit une fois par mois pour évaluer les activités passées et pour assurer le suivi des projets en cours. Il est directement responsable devant l'assemblée générale, est responsable de la distribution des terres au sein de la communauté et est structuré par des commissions;

- L'unité de gestion du projet (ou union), qui est composée de 6 membres choisis sur une base paritaire (3 de chaque village) et nommés par le conseil. Elle se réunit une fois tous les 15 jours et gère le projet à l'échelle locale. Elle est nommée pour un mandat de trois ans.

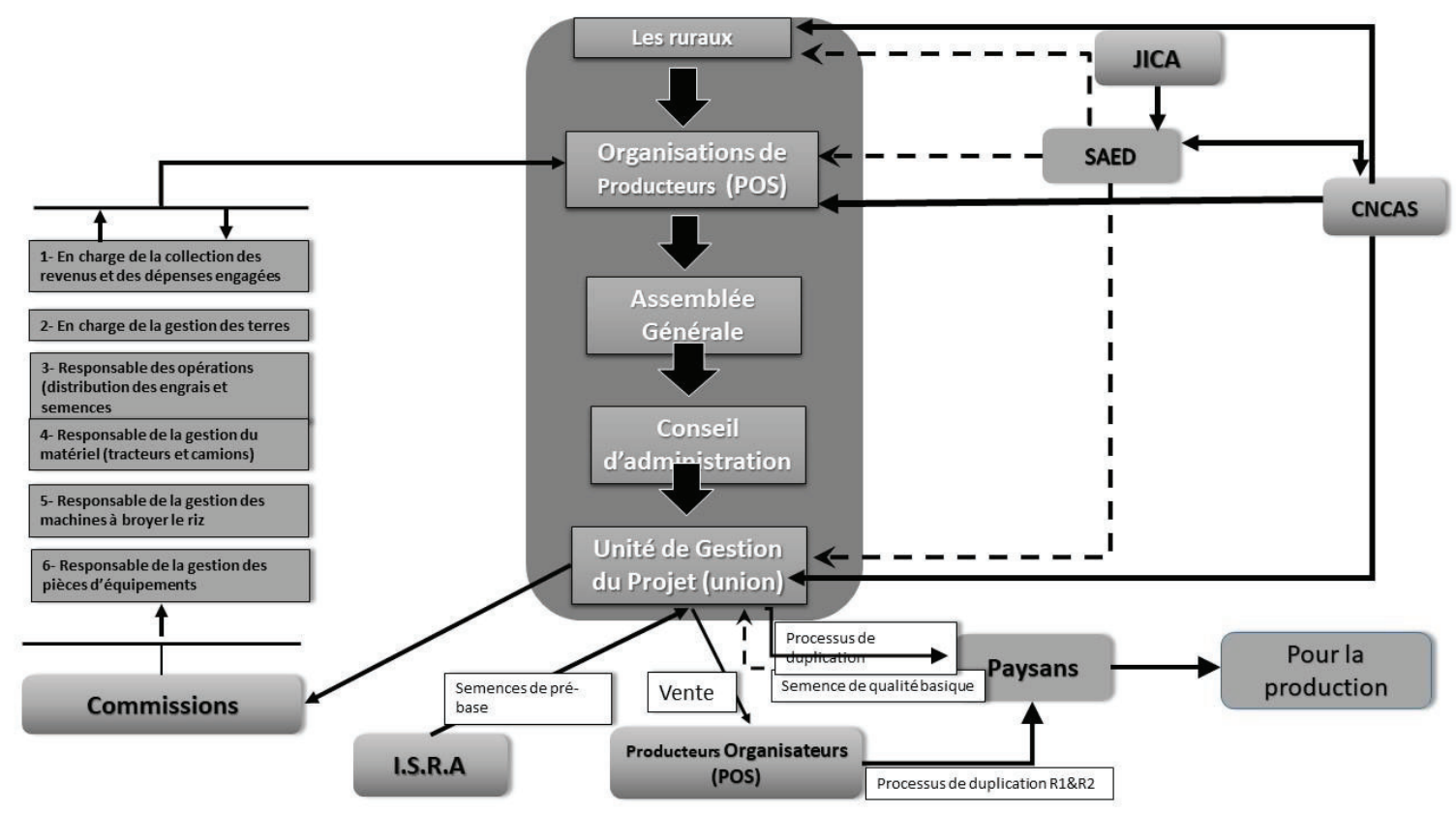

Figure 11 - Cadre institutionnel du projet d'irrigation Débi-Tiguet Source : Mbaye, 2018

Enfin, les différentes commissions du conseil d'administration sont chacune gérées par un commissaire et par un commissaire adjoint :

- la commission chargée de percevoir les recettes et d'engager les dépenses;

- la commission chargée de la gestion des terres;

- la commission chargée de l'exploitation (distribution de semences et d'engrais);

- la commission chargée de la gestion des équipements (tracteurs et camions);

- la commission chargée de la gestion des machines à décortiquer le riz;

- la commission chargée de la gestion des pièces d'équipement.
Chaque commission présente un rapport de ses activités lors de chaque réunion. Chaque OP se voit attribuer une parcelle de terrain donnée, évalue ses besoins et prépare une demande de prêt à la Caisse nationale de crédit agricole du Sénégal (CNCAS). La SAED évalue la faisabilité technique et financière de la demande avant de donner son accord à la CNCAS pour que le prêt soit approuvé. La SAED suit les déboursements des prêts avec les OP. La demande de prêt est personnelle ; elle met chaque producteur en contact avec la CNCAS, même si le processus est collectif. Il est à noter que les OP ne reçoivent pas d'argent liquide, mais des bons qui peuvent être échangés contre des semences, des engrais ou tout autre intrant dont 
elles peuvent avoir besoin dans le processus de production. Avant d'accorder des prêts, la banque demande un dépôt correspondant au quart des besoins financiers, en s'assurant que chaque OP dispose d'un dépôt à la banque (Mbaye,2008).

Les récoltes sont centralisées auprès de la commission et chaque dépôt est certifié par un titre. La commission (par le biais de sa commission responsable) décortique le riz, vend la production, rembourse la banque en fonction de la part de chaque producteur et reverse le solde aux OP.

L'union facture aux producteurs divers services aux taux suivants :

- Eau irriguée : 62500 francs CFA par ha;

- Gestion des techniques agricoles : 19000 francs CFA par ha;

- Semences certifiées : 250 francs CFA par kg;

- Les camions (quatre de 5 tonnes de charge utile et un de 10 tonnes de charge utile) sont loués aux OP. Les coûts facturés sont de 25000 francs CFA par jour; mais des rabais exceptionnels sont accordés pour certaines activités particulières. Par exemple, le prix demandé pour la machine de décorticage est de 10000 francs CFA, et de 5000 francs CFA pour les activités opérationnelles. En ce qui concerne le camion de 10 tonnes, il est affecté à la livraison des engrais, des semences et des récoltes, et les coûts facturés pour ces services ne sont que de 5000 francs CFA par jour. Les camions peuvent être loués par des personnes en dehors de l'union pour un usage privé. Dans ce cas, la commission chargée de sa gestion détermine de manière discrétionnaire le montant à facturer;

- Le décorticage du riz : 13 francs CFA par kg.

Tous ces services sont également accessibles aux opérateurs privés qui cultivent du riz dans les environs de Débi-Tiguet, mais à un prix plus élevé que ce que paient les OP (Mbaye, 2008). Chaque commission détermine sa marge en comparant les recettes aux coûts. Ces dernières années, les coûts ont eu tendance à dépasser les recettes; les frais de service n'ont pas augmenté au cours des 10 dernières années, à l'exception des frais de localisation des tracteurs, qui ont augmenté de 1500 francs CFA, tandis que les frais d'eau ont baissé de
5000 francs CFA au cours de la même période et les coûts de décorticage, de 1 franc CFA par kg.

Les recettes sont utilisées par l'union de la manière suivante :

- $44 \%$ pour la gestion de la station de pompage : essence pour le drainage et pour les stations d'irrigation, pièces d'équipement, entretien (service effectué par la SAED et facturé à l'union), etc.;

- $41 \%$ pour la prise en charge de l'exploitation (distribution de semences et d'engrais);

- $15 \%$ du produit total déposé à la banque dans un compte d'épargne afin de s'assurer contre les risques inattendus; toutefois, aucun risque de ce type ne s'est présenté au cours des 10 dernières années. La valeur actuelle du dépôt est de 147 millions de francs CFA.

La coopération avec l'Institut sénégalais de recherche agricole (ISRA) est également jugée satisfaisante. L'ISRA produit des semences de qualité de base et les vend à l'union au prix de 720 francs CFA par kg. Ensuite, l'union choisit les paysans qui ont le mieux démontré leurs capacités à suivre l'itinéraire technique tracé par la recherche pour dupliquer les semences. Ces semences produites par les paysans sont de qualité de base et coûtent 175 francs CFA par $\mathrm{kg}$. En contrepartie, l'union les vend au prix de 350 francs CFA après avoir pris en charge les frais d'emballage, de tri, de transport, etc. aux OP. L'union réalise une marge bénéficiaire estimée à 20 francs CFA. L'étape finale est que les OP dupliquent encore ces semences aux niveaux R1 et R2, puis les distribuent aux paysans pour la production.

\section{Conclusion}

L'objectif de cet article était d'analyser les modèles d'organisation de la zone de production de riz, et comment ils affectent la dynamique de l'emploi et de la formation des revenus. À partir des données d'EJMAO, nous avons analysé les caractéristiques sociodémographiques des organisations paysannes dans la vallée du fleuve Sénégal. Le cas des organisations paysannes des villages de Débi et Tiguet représente une bonne illustration d'innovation sociale pour accroitre le potentiel d'emploi dans ces zones agricoles et pour augmenter le revenu des organisations de producteurs. 


\section{NOTE}

$112600 €$ ou $19500 \$ C A$.

\section{RÉFÉRENCES}

Benjamin, N. et Mbaye, A. A. (2012). Informality, productivity and enforcement in West Africa: A firm-level analysis. Review of Development Economics, 16(4), 664-680. https://doi.org/10.1111/rode.12010

Defoer, T., Wopereis, M., Jones, M. P., Lanson, F., Erenstein, O. et Guei, R. G. (2004, février). Challenges and technical opportunities for rice-based production system for food security and poverty alleviation in Sub-Saharan Africa. Communication présentée à la FAO Rice Conference de la West Africa Rice Development Association, Rome.

Repéré à http://www.fao.org/rice2004/en/pdf/warda.pdf

Dingkuhn, M. et Sow, A. (1997). Potential yields of irrigated rice in the Sahel. Dans K. M. Miézan, M. Wopereis, M. Dingkuhn, J. Deckers et T. F. Randolph (dir.), Irrigated rice in the Sabel: Prospects for sustainable development (p. 361-380). West Africa Rice Development Association. Repéré à https:/ / agritrop.cirad.fr/309422

Initiative Prospective Agricole et Rurale (IPAR) (2015). Emploi des jeunes et migration en Afrique de l'Ouest (EJMAO) - Rapport final. Repéré à https://idl-bnc-idrc.dspacedirect.org/bitstream/handle/10625/54153/IDL-54153.pdf

Fall, A. et Cissé, A. (2010). Diagnostic de la filière riz dans le bassin de l'Anambé et anabyse de la situation socio-économique des membres de la FEPROBA. VECO.

Food and Agriculture Organization of the United Nations (FAO). (2009). Rice price update: Commodity markets, policy analysis and projections service. FAO.

Golub, S. et Mbaye, A. A. (2015). Creating good jobs in Africa: Demand- and supply-side policies. Jobs and Development Blog. Repéré à https://works.swarthmore.edu/fac-economics/371

FERT et IECD. (2015). Former les jeunes ruraux : les conditions d'une insertion réussie. Grain de sel, 71, 24-25. Repéré à https://www.inter-reseaux.org/IMG/pdf/gds71_complet.pdf

Mbaye, A. A., Beye, A., Gueye, A., Lokonon, B. et Ndione, Y. C. (2016). Generating employment and increasing income in agricultural value chains thereby fostering food security: Case studies of rice and cotton in Benin and Senegal. Discussion paper on development policy no. 254. ZEF. Repéré à https://www.researchgate.net/publication/342766002_Generating_employment_and_ increasing_income_in_agricultural_value_chains_and_thereby_fostering_food_security_Case_studies_of_rice_and_ cotton_in_Benin_and_Senegal

Mbaye, A. A., Golub, S. et Gueye, F. (2020). Formal and informal enterprises in francophone Africa: Moving toward a vibrant private sector. Ottawa, Ontario : CRDI. Repéré à https://www.idrc.ca/fr/livres/formal-and-informal-enterprises-francophone-africa-moving-towardvibrant-private-sector

Mbaye, A. A. (2008). Aid Effectiveness to Infrastructure: A Comparative Study of East Asia and Sub-Saharan Africa: Senegal Case Study. JBICI Research Paper No. 36-3. Repéré à https://www.jica.go.jp/jica-ri/IFIC_and_JBICI-Studies/ jica-ri/research/archives/jbic/pdf/rp36_e08.pdf

Programme National d'Autosuffisance en Riz (PNAR). République du Sénégal, Ministère de l'Agriculture (2009). Repéré à https://www.ipar.sn/IMG/pdf/2009_Prog_Nat_Autosufisance_Riz.pdf

République du Sénégal. (2001). Rapport d’analyse : Enquête sénégalaise auprès des ménages (2e éd.). Dakar, Sénégal. Repéré à http://anads.ansd.sn/index.php/catalog/47

Société d'aménagement et d'exploitation des terres du Delta du fleuve Sénégal et des vallées du fleuve Sénégal et de la Falémé (SAED). (2011). Recueil de la vallée du fleuve Sénégal 2008-2009. Dakar, Sénégal.

Société Nationale d'Aménagement et d'Exploitation des Terres du Delta du Fleuve et des Vallées du Fleuve Sénégal (SAED) (2003). L'expérience du Groupe Import-Export Khady Sao Secke sur la Commercialisation du riz dans la vallée du Fleuve Sénégal. Dakar, Sénégal.

Wopereis, M., Donovan, C., Nebie, B., Guindo, D. et Ndiaye, M. (1999). Soil fertility management in irrigated rice systems in the Sahel and savanna regions of West Africa (1 1e $^{\text {pe }}$ partie : Agronomic Analysis). Field Crops Research, 61(2), 125-144.

https://doi.org/10.1016/S0378-4290(98)00154-3 